\title{
A New Error in Variables Model for Solving Positive Semi-Definite Linear Systems with Applications to Well-known Control Problems
}

\author{
Negin Bagherpour ${ }^{1}$, Nezam Mahdavi-Amiri \\ ${ }^{1}$ Department of Mathematical Sciences, The University of Manchester \\ Manchester, UK \\ Negin.bagherpour@manchester.ac.uk \\ ${ }^{2}$ Department of Mathematical Sciences, Sharif University of Technology \\ Tehran, Iran \\ Nezamm@sharif.ir
}

\begin{abstract}
We have recently presented a competitive method to solve overdetermined linear systems of equations with multiple right hand side vectors, where the unknown matrix is symmetric and positive definite. The coefficient and the right hand side matrices are respectively named as data and target matrices. There are also physical contexts, such as modeling a deformable structure and computing the correlation matrix in finance or insurance/reinsurance industries, where a symmetric positive semi-definite solution of an overdetermined linear system of equations needs to be computed. Several methods have been proposed for solving such problems with the assumption that the data matrix is unrealistically error free. In real measurements, however, both the data and target matrices may contain error. Thus, for practical purposes, an error in variables model appears to be more appropriate. Here, we define a new error function to consider error in both data and target matrices and propose an iterative algorithm to minimize the defined error. We illustrate that our proposed approach turns to be efficient in attaining accurate solutions. For this purpose, we generate random test problems and show that our proposed algorithm computes the solution faster than the existing methods. Moreover, the standard deviation in error matrix is considerably lower when we use our proposed algorithm. This feature makes our method more efficient and desirable in real applications.
\end{abstract}

Keywords: Semi-definite linear systems, Error in variables model, Correlation matrix computing, Minimum rank problem

\section{Introduction}

In several physical problems, such as estimation of the mass inertia matrix in design of controllers for solid structures and robots, an overdetermined linear system of equations with multiple right hand side vectors arises with the constraint that the unknown matrix be symmetric and positive definite. We have recently introduced a method for solving such a problem, using a newly defined error in variables (EIV) model; see [3]. There are also physical contexts, such as modeling a deformable structure [1] and computing the correlation matrix in finance or insurance/reinsurance industries [2], where a symmetric positive semi-definite solution of an overdetermined linear system of equations needs to be computed or equivalently, the problem

$$
D X \simeq T
$$

is needed to be solved, with $D, T \in \mathbb{R}^{m \times n}, \mathrm{~m} \geq \mathrm{n}$, are given and $X \in \mathbb{R}^{n \times n}$, a symmetric positive semi-definite matrix, is to be computed as a solution. Available methods for solving such a problem consider an ordinary least squares formulation; e.g., see [1]. A practically useful error in variables (EIV) model was introduced in [3] for a positive definite linear system of equations. Based on this formulation, with $(\cdot)$ standing for trace of a matrix, a solution of the optimization problem

$$
\min _{X \succ 0} \operatorname{tr}\left((D X-T)^{\mathrm{T}}\left(D-T X^{-1}\right)\right)
$$


is a solution of a corresponding positive definite linear system of equations, where $X$ is symmetric and by $X>0$, we mean $X$ is positive definite. The error formulation in [3] not being suitable here, we first motivate and present a new error formulation for the positive semi-definite case.

In (2), the entries of $D-T X^{-1}$ and $D X-T$ represent the errors in $D$ and $T$, respectively. Here, we need to find a represent the error in $D$ without engaging $X^{1}$. Before describing the EIV model to solve the positive semi-definite linear system of equations, we consider a newly noted problem, the positive semi-definite linear system of equations with a given rank $r$ of the unknown matrix (here, denoted by PSDEIV-R $r$ ).

\section{Error Formulation}

The error in $D$ is supposed to be the difference between the real value of $D$ and the predicted value for $D$ obtained by $D X \simeq T$. To compute the predicted value for $D$, we use the general least squares solution of the system $X D^{\mathrm{T}} \simeq T^{\mathrm{T}}$. Considering the block forms $D^{\mathrm{T}}=\left(d_{1}, \ldots, d_{m}\right)$ and $T^{\mathrm{T}}=\left(t_{1}, \ldots, t_{m}\right)$; where $d_{i}, t_{i} \in \mathbb{R}^{n}$, for $i=1, \ldots, m$, we have $X d_{i}=t_{i}$, for $i=$ $1, \ldots, m$. The general solution to such a linear system is

$$
d_{i}=X^{\dagger} t_{i}+n_{i}
$$

where $X^{\dagger}$ is the pseudo-inverse of $X$ and $n_{i}$ is an arbitrary vector in the null space of $X$ [4]. A straightforward choice for $n_{i}$ is $n_{i}=0$, which results in $d_{i}=X^{\dagger} t_{i}$ and $\Delta D=D-T X^{\dagger}$. We later consider suitable choices for $n_{i}$ which minimize $\|\Delta D\|_{\mathrm{F}}$, where $F$ stands for the Frobenius norm. Note that to compute $d_{i}$ from (3), the spectral decomposition can be applied.

Now, making use of the spectral decomposition of $X$ in (3), we can write the general solution as $d_{i}=X^{\dagger} t_{i}+U_{n-r} z_{i}$; where $z_{i} \in \mathbb{R}^{n-r}$, for $i=1, \ldots, m$, are arbitrary vectors. Thus, $D^{T}=X^{\dagger} T^{T}+U_{n-r}\left(z_{1} \ldots z_{m}\right)$; and the predicted value for $D$ is

$$
D_{p}=T X^{\dagger}+Z U_{n-r}^{\mathrm{T}}
$$

where $Z \in \mathbb{R}^{m \times(n-r)}$ is arbitrary, and the error in $D$ is $\Delta D=\mathrm{D}-\left(T X^{\dagger}+Z U^{\mathrm{T}}{ }_{n-r}\right)$. A reasonable choice for $Z$ in this formulation would be the one minimizing the norm of $\Delta D$, which is given as the solution of the optimization problem

$$
\min _{Z}\left\|F-Z U_{n-r}^{\mathrm{T}}\right\|_{\mathrm{F}}^{2}
$$

where $F=D-T X^{\dagger}$. Solving (5) results in [4]

$$
Z^{*}=F U_{n-r}=\left(D-T X^{\dagger}\right) U_{n-r}
$$

Substituting (6) in (4), we get

$$
\begin{aligned}
\Delta D & =D-\left(T X^{\dagger}+Z^{*} U_{n-r}^{\mathrm{T}}\right)=\left(D-T X^{\dagger}\right)-\left(D-T X^{\dagger}\right) U_{n-r} U_{n-r}^{\mathrm{T}} \\
& =\left(D-T X^{\dagger}\right)\left(I-U_{n-r} U_{n-r}^{\mathrm{T}}\right) \\
& =\left(D-T X^{\dagger}\right) U_{r} U_{r}^{\mathrm{T}} .
\end{aligned}
$$

Based on the above discussion, $\Delta D=\left(D-T X^{\dagger}\right) U_{r} U_{r}^{\mathrm{T}}$ and $\Delta T=D X-T$ respectively represent the errors in $D$ and $T$. Thus, to solve a rank $r$ positive semi-definite linear system of equations, it is appropriate to minimize the error

$$
E=\sum_{i=1}^{m} \sum_{j=1}^{n} \Delta D_{i j} \Delta T_{i j}=\operatorname{tr}\left(\Delta T^{\mathrm{T}} \Delta D\right) .
$$

Therefore, the following problem is posed: 


$$
\begin{array}{cl}
\min & \operatorname{tr}\left(\Delta T^{\mathrm{T}} \Delta D\right) \\
\text { s.t. } & X \succeq 0 \\
& \operatorname{rank}(X)=r .
\end{array}
$$

Using the spectral decomposition of $X$, we get

$$
E=\operatorname{tr}\left(U_{r}^{\mathrm{T}} A U_{r} S^{2}-U_{r}^{\mathrm{T}} C U_{r}+U_{r}^{\mathrm{T}} B U_{r} S^{-2}\right),
$$

where $A=D^{\mathrm{T}} D, C=D^{\mathrm{T}} T+T^{\mathrm{T}} D$ and $B=T^{\mathrm{T}} T$. Hence, the solution $X$ is computed by letting $X=Y^{*}\left(S^{*}\right)^{2}\left(Y^{*}\right)^{\mathrm{T}}$ in which $Y^{*} \in$ $\mathbb{R}^{n \times r}$ and $S^{*}=\operatorname{diag}\left(s_{1}, s_{2}, \ldots, s_{r}\right)$ form a solution of

$$
\begin{array}{cl}
\min & F(Y, S), \\
\text { s.t. } & Y^{\mathrm{T}} Y=I \\
& s_{i} \neq 0, \quad i=1, \cdots, r,
\end{array}
$$

with $F(Y, S)=\left(Y^{T} A Y S^{2}-Y^{T} C Y+Y^{T} B Y S^{-2}\right)$.

Next, we discuss how to solve (11). We are to develop an iterative algorithm by first solving the following related problem:

$$
\begin{array}{cl}
\min & \operatorname{tr}\left(Y^{\mathrm{T}} A Y S^{2}-Y^{\mathrm{T}} C Y+Y^{\mathrm{T}} B Y S^{-2}\right) \\
\text { s.t. } & S=\operatorname{diag}\left(s_{1}, \cdots, s_{r}\right),
\end{array}
$$

for a given $Y \in \mathbb{R}^{n \times r}$ in each iteration and then use the results to solve (11).

The solution to (12) for an arbitrary $Y \in \mathbb{R}^{n \times r}$ is given by $S=\operatorname{diag}\left(s_{l}, \cdots, s_{r}\right)$, with

$$
s_{i}= \pm\left(\frac{y_{i}^{\mathrm{T}} B y_{i}}{y_{i}^{\mathrm{T}} A y_{i}}\right)^{\frac{1}{4}}, \quad i=1, \cdots, r
$$

where $y_{i}$ is the $i$ th column of $Y$. Note that the above solution leads to a unique value of $S^{2}$ :

$$
S^{2}=\operatorname{diag}\left(\left(\frac{y_{i}^{\mathrm{T}} B y_{i}}{y_{1}{ }^{\mathrm{T}} A y_{1}}\right)^{\frac{1}{2}}, \cdots,\left(\frac{y_{i}^{\mathrm{T}} B y_{i}}{y_{r}{ }^{\mathrm{T}} A y_{r}}\right)^{\frac{1}{2}}\right) \text {. }
$$

\subsection{Mathematical Solution}

We can now proceed to solve (11). For a given $S$, we consider an optimization problem, equivalent to (11), which is simpler to solve. Let $Y \in V_{\mathrm{r}}\left(\mathbb{R}^{n}\right)$ be a given matrix and the diagonal matrix $S(Y)$ be computed by (13). The optimization problem

$$
\begin{array}{cl}
\min & F(Y, S(Y)) \\
\text { s.t. } & Y \in V_{r}\left(\mathbb{R}^{n}\right),
\end{array}
$$

is equivalent to (11), meaning that both (11) and (14) have the same Karush-Kuhn-Tucker (KKT) points. To find a KKT point for the optimization problem (11), we solve (14) by using a proper optimization algorithm on the Stiefel manifold, that is, the superlinearly convergent BFGS algorithm of [5] for solving

$$
\begin{array}{cl}
\min & F(X), \\
\text { s.t. } & X \in V_{r}\left(\mathbb{R}^{n}\right) .
\end{array}
$$

As discussed in [5] and [6], PSDEIV-Rr converges globally to a local solution of (11) with an asymptotic superlinear convergence rate. 
To extend our proposed method for solving positive semi-definite linear system of equations over all ranks, we solve the problem presuming all possible ranks to attain a solution with a minimal error. We further adapt the proposed approach to solve two well-known problems in control theory, the minimum rank problem and the problem of computing correlation matrix as explained next.

(i) Positive semi-definite EIV minimum rank problem:

$$
\begin{array}{cl}
\min & \operatorname{rank}(X) \\
\text { s.t. } & \operatorname{tr}\left(\Delta D^{\mathrm{T}} \Delta T\right)<e \\
& X \succeq 0 .
\end{array}
$$

We start from the smallest nontrivial possible rank, $r=1$, and solve the corresponding positive semi-definite linear system of equations to compute the solution $X_{r}$ and the corresponding EIV error $E(r)$. If the inequality $E(r)<e$ holds for the computed $X_{r}$, then we stop; otherwise, we increase $r$ by one and continue iteratively until satisfying the inequality $E(r)$ $<e$. The number of iterations may be at most $n$. Note that if after $\mathrm{n}$ iterations, none of the matrices $X_{r}, r=1, \cdots n$, satisfies the inequality $E(r)<e$, then we may consider $X_{r}$ with the smallest corresponding value $E(r)$ as an approximate solution of the minimum rank problem.

(ii) Computing the correlation matrix: This problem is a special case of the positive semi-definite linear system of equations. Computing a correlation matrix is equivalent to finding a positive semi-definite matrix $X$ to satisfy $X=C$ and $P X=Q$. The linear constraints $X=C$ and $P X=Q$ can be replaced by the overdetermined system of equations

$$
\left(\begin{array}{l}
I \\
P
\end{array}\right) X=\left(\begin{array}{l}
C \\
Q
\end{array}\right) \text {. }
$$

To solve the overdetermined system of equations (16), an ordinary formulation has been considered [2]. We note that (16) is a special case of the positive semi-definite linear system of equations with data and target matrices $D=\left(\begin{array}{l}I \\ P\end{array}\right)$ and $\mathrm{T}=$ $\left(\begin{array}{l}T \\ Q\end{array}\right)$, where $I$ is the $n \times n$ identity matrix, $P, Q \in \mathbb{R}^{m \times n}$ and $C \in \mathbb{R}^{n \times n}$ are arbitrary.

\section{Numerical Results}

Comparative numerical results show the efficiency and competitiveness of our proposed algorithms. Also, comparisons of the results obtained by our proposed approaches for solving positive semidefinite linear systems of equations, minimum rank problem and the problem of computing correlation matrix with some available methods confirm competetiveness of our algorithms in computing solutions with lower values of standard deviation more efficiently. The Dolan-More performance' profiles also demonstrate both the efficiency and accuracy of our proposed methods.

\section{Conclusion}

We proposed a new approach to solve positive semi-definite linear system (PSDLS) of equations. Our proposed error estimate for both data and target matrices admitted a realistic problem formulation. We first considered a newly defined problem, namely the given rank positive semi-definite linear system (Rr-PSDLS) of equations, and presented a globally convergent algorithm for its solution. The proposed algorithm also has an asymptotic superlinear rate of convergence. Numerical results confirmed the effectiveness of our approach to compute solutions of R $r$-PSDLS more efficiently than the trust region code in MANOPT toolbox of MATLAB. We further showed how to apply the error in variables (EIV) model for solving rank $r$ positive semi-definite linear system of equations (PSDEIV-Rr) to solve the general PSDLS. Based on the obtained numerical results, our method for solving the PSDLS showed to be more efficient than an interior point method and a path following algorithm. We also introduced novel algorithms based on the proposed EIV method to solve two wellknown problems in control theory: minimum rank and correlation matrix estimation. The numerical results confirmed the efficiency and accuracy of the algorithms in comparison with the available methods. 


\section{References}

[1] N. G. Krislock, J. Lang, J. Varah, D. K. Pai and H. Seidel, "Local compliance estimation via positive semi-denite constrained least squares," IEEE Trans. Robotics and Automation, vol. 20, no. 6, pp. 1007-1011, 2004.

[2] D. Sun and Y. Gao, "Calibrating least squares covariance matrix problems with equality and inequality constraints," SIAM J. Matrix Anal. Appl., vol. 31, pp. 1432-1457, 2009.

[3] N. Bagherpour and N. Mahdavi-Amiri, "A new error in variables model for solving positive definite linear system using orthogonal matrix decompositions," Numer. Algor., vol. 72, no. 1, pp. 211-241 2016.

[4] C. F. Van Loan and G. Golub, Matrix Computations, 4th ed. JHU Press, 2012.

[5] W. Huang, K. A. Gallivan and P.-A. Absil, "A Broyden Class of Quasi-Newton Methods for Riemannian Optimization,” SIAM J. Optim., vol. 25, no. 3, pp. 1660-1685, 2015.

[6] M. F. Anjos, N. J. Higham, P. L. Takouda and H. Wolkowicz, "A semidefinite programming approach for the nearest correlation matrix problem," Technical Report, Combinatorics and Optimization, University of Waterloo, 2003.

[7] K. C. Toh. (2009, April 10). QSDP version beta - a MATLAB software for convex quadratic semidefinite programming. [Online]. Available: http://www.math.nus.edu.sg/ mattohkc/qsdp.html 